\title{
The Plasma Treatment Unit An Attempt to Standardize Cold Plasma Treatment for Defined Biological Effects
}

\author{
Alexander Fridman, ${ }^{a}$ Abraham Lin, ${ }^{\mathrm{b}}$ Vandana Miller, ${ }^{\mathrm{a}, *}$ Sander Bekeschus, ${ }^{\mathrm{c}}$ \\ Kristian Wende, ${ }^{c}$ Klaus-Dieter Weltmann ${ }^{c}$ \\ aDrexel University, Nyheim Plasma Institute, Camden, New Jersey, USA; 'University of Antwerp, \\ Department of Chemistry, Antwerp, Belgium; 'Leibniz-Institute for Plasma Science and \\ Technology (INP Greifswald), ZIK Plasmatis, Greifswald, Germany \\ *Address all correspondence to: Vandana Miller, 200 Federal Street, Suite 500, Camden, NJ 08103, USA; Tel.: 215 - \\ 571-4074, E-mail: vam54@drexel.edu
}

\begin{abstract}
Plasma bioscience and medicine are both rapidly growing fields. Their aim is to utilize cold physical plasmas for desired biological outcomes in medicine, biotechnology, agriculture, and general hygienic purposes. Great success has been achieved in many applications with individually designed plasma sources and plasma parameters. Although lab and application-specific tuning of plasmas is a great advantage of this technology, standardized units to define plasma treatments are required to facilitate comparison of the effects found by different researchers who do not use the same plasma sources. By drawing conclusions from over a century of plasma biomedical research, we propose that all researchers adopt the use of a standardized value, the plasma treatment unit (PTU), to describe the biological effects of different cold plasma sources and treatment regimens. It quantifies a key plasma effector in biological systems as an indicator and may provide the foundation for an analogous and clinically relevant unit in the future.
\end{abstract}

KEY WORDS: plasma medicine, nonequilibrium atmospheric pressure plasma, cold atmospheric plasma, dielectric barrier discharge, kINPen

\section{STANDARDIZATION CHALLENGE FOR PLASMA MEDICINE}

As our understanding of non-equilibrium, atmospheric pressure plasma, also known as cold plasma, improves, plasma delivery devices for biological and medical applications are becoming increasingly more sophisticated. Their application on various biological systems, such as prokaryotic cells, viruses, and eukaryotic cells (yeast, plant, and mammalian), has broadened and enhanced the identification of the complex interplay between plasma and cellular processes. Based on the results of in vitro and in vivo studies to explore the biological, biochemical, and genetic changes in tissues as well as organismal changes that are initiated by plasmas, it is now recognized that plasma effects are largely a result of oxidation-reduction (redox) changes produced by plasma components. These include reactive oxygen species (ROS), reactive nitrogen species (RNS), charged species, electric fields, and ultraviolet (UV) light, and the observed outcomes are most likely due to a combinatorial effect. These range from proliferation to differentiation and all the way to the death of both prokaryotic and eukaryotic cells, as a function of plasma parameters and treatment conditions. These in vitro observations have provided the impetus to test 
plasmas in experimental and clinical settings for treatment of many conditions, including cancers and chronic wounds, within the field of plasma medicine. ${ }^{1-6}$

Amid increasing interest in plasma technology from biologists and clinicians, several plasma sources are now available for these applications. These fall into two major categories: dielectric barrier discharge (DBD), where plasma is generated directly on the target; and the atmospheric pressure plasma jet, where remotely generated plasma is transported to the target through flow of an inert gas..$^{7-12}$ The plasma produced by both DBDs and jets may be changed by altering the properties of the applied electrical fields and the treatment surface (e.g., conductivity and surface uniformity). Plasmas produced by plasma jets may also be varied by changing the flow rate and composition of the working gas. For DBDs, the distance between the high-voltage electrode and treatment target is an important determinant of the composition of plasma (Table 1). However, plasmas produced by plasma jets are more stable than those produced by DBDs because the discharge environment can be tightly controlled by the feed gas. ${ }^{13}$ DBDs are generated in atmospheric air and often depend on target properties, so variations in environmental conditions, such as humidity and skin conductivity may influence the plasma discharge and subsequent biological outcomes. ${ }^{14,15}$

Both DBDs and plasma jets have their individual advantages and disadvantages in the clinical setting. DBD electrodes can be made in different sizes for precise treatments or large field treatments. ${ }^{6,16-18}$ On the other hand, plasma jets are often limited to spot treatments, because the plasma plume from the jet aperture is only a few millimeters in diameter. ${ }^{19}$ Treatment of large areas, as is in the case of burns and wounds, requires sweeping the jet over the entire surface, which could take considerable time. ${ }^{3,13,19}$ Because DBDs use the patient as the second electrode, they are limited by more stringent electrical safety measures.

The final composition of plasma between these two sources differs only slightly, and the overall observed biological effects are similar, though not identical. Both DBDs and plasma jets have been shown to improve clinical outcomes for wound healing and in cancer treatment. Although there are several overlapping parameters that both plasma systems rely on, such as treatment time, frequency, and voltage (see Table 1), the impact of each parameter on biological effect varies between devices. A 1-minute treatment with a plasma jet rarely produces the same effect as a 1-minute treatment with a DBD. This lack of standardized plasma treatment between devices poses significant complications for the clinician, and it hinders wider clinical acceptance. Establishment of a com-

TABLE 1: Factors that influence treatment outcome with DBDs and plasma jets

\begin{tabular}{|l|l|l|}
\hline & DBD & Plasma jet \\
\hline Excitation & Pulsation, pulse shape & Pulsation \\
\hline Plasma parameters & Voltage, frequency, & Voltage, frequency \\
\hline Treatment parameters & $\begin{array}{l}\text { Treatment time, application } \\
\text { distance }\end{array}$ & $\begin{array}{l}\text { Treatment time, application } \\
\text { distance }\end{array}$ \\
\hline Discharge environment & Atmospheric gas, humidity & $\begin{array}{l}\text { Type of feed gas, humidity, flow } \\
\text { rate }\end{array}$ \\
\hline
\end{tabular}


mon treatment unit for these plasma devices would promote acceptance and ease clinical adoption of this technology.

A recently published study used a single parameter to characterize a predefined biological expectation from the use of a DBD and a plasma jet. ${ }^{20}$ The goal was to operate both plasma units to achieve the same biological outcomes under standardized laboratory conditions. A reinterpretation of the results of that study may propose a simple and cheap method of establishing a plasma treatment unit (PTU) that can be used to correlate treatment with DBDs and plasma jets for comparable device calibration.

\section{A. Defining the Plasma Treatment Unit}

Each plasma device requires a precise combination of several operating parameters to achieve a specific biological effect. Therefore, if a shared PTU for these devices is to become practical and easily adapted, it should not depend on any of the physical parameters of individual devices. Rather, the basis of the PTU should be a biological readout, because the ultimate goal is for the devices to yield reproducible clinical effects. Bekeschus et al. suggest the use of cellular metabolic activity, a reflection of plasma-induced toxicity, identified with a simple and concise live-cell metabolic activity assay. ${ }^{20}$ This is analogous to the wellestablished approach in determining dosage of therapeutic chemicals where the initial titration is done in vitro, and then clinical end points are used to guide in vivo administration. To compare plasma systems with this assay, it is necessary that procedures for cell plating and readouts for 50\% inhibition of metabolic activity (IC50) be strictly defined (Table 2).

Using their observations from the standardized conditions listed here, we define 1 PTU as the IC50 of cells, as measured with the live cell metabolic activity assay. This means that the user is free to operate their device at any physical parameters they wish, as long as the biological outcome is the same (i.e., 50\% inhibition of cellular metabolic activity). This assay is quite stringent, requires aseptic laboratory procedures, and may not be practical for calibration of devices to produce comparable effects. However, if it is translated into a more basic, easily performed assay, this calibration may be possible.

The effectors in plasma include several ROS, RNS, electric fields, and UV light. Hydrogen peroxide $\left(\mathrm{H}_{2} \mathrm{O}_{2}\right)$ is one of the molecules produced by both DBDs and plasma jets used in clinical and biological research settings. ${ }^{21}$ It is easily measured using a simple and

TABLE 2: Fixed parameters and procedures for standardization

\begin{tabular}{|l|l|}
\hline Parameters to define & \\
\hline Seeding time & 24 hours before plasma treatment \\
\hline Seeding concentration & $3 \times 10^{5}$ cells $/ \mathrm{mL}$ \\
\hline Treatment chamber & 24 -well plates $(0.5 \mathrm{~mL} /$ well $)$ \\
\hline Liquid present during treatment & Phosphate buffered saline \\
\hline Volume of liquid present & $1000 \mu \mathrm{L}$ or less with dilution factor \\
\hline Incubation time prior to live cell metabolic activity assay & 24 hours post-plasma treatment \\
\hline
\end{tabular}

Volume 8, Issue 2, 2018 
inexpensive colorimetric assay. Therefore, at the plasma device settings corresponding to $1 \mathrm{PTU}, \mathrm{H}_{2} \mathrm{O}_{2}$ production by both plasma systems was measured and compared. Nanosecond-pulsed DBD (nspDBD) and kINPen (neoplas tools GmbH, Walther-Rathenau-Str. 49a, 17489 Greifswald, Germany) generated $50 \mu \mathrm{M}$ and $115 \mu \mathrm{M} \mathrm{H}_{2} \mathrm{O}_{2}$ respectively, at their specific 1 PTU operating parameters. Although this indicates the desired metabolic inhibition was achieved by different mechanisms, device calibration is an easier process.

\section{B. Defining $\triangle$ PTU}

A logical question that arises is how to scale this unit? From a physics perspective, 2 PTU is not achieved by doubling a single parameter such as treatment time or frequency. From a biological perspective, 2 PTU is not equivalent to doubling the biological effect (IC100). Therefore, 2 PTU is perhaps best defined as a repetition of the plasma physics (operating parameters) that were established to get the effect of 1 PTU. This encompasses all the parameters needed to produce the plasma that elicits IC50.

An extrapolation to the 2 PTU scenario can be made using the $\mathrm{H}_{2} \mathrm{O}_{2}$ measurement example just mentioned. Two PTU would encompass the settings that elicit twice the $\mathrm{H}_{2} \mathrm{O}_{2}$ for each device: $100 \mu \mathrm{M}$ for an nspDBD and $230 \mu \mathrm{M}$ for a kINPen. Therefore, PTU need not scale according to a single plasma parameter, such as duration of treatment or pulse repetition; rather, it accounts for and encompasses all device variables as measured by an indicator molecule, which in this case was $\mathrm{H}_{2} \mathrm{O}_{2}$. This does not suggest that $\mathrm{H}_{2} \mathrm{O}_{2}$ is the only factor responsible for the measured biological effect. It only indicates that the conditions under which a measured biological effect was produced corresponds to a specified amount of $\mathrm{H}_{2} \mathrm{O}_{2}$ produced. In fact, plasma generated significantly less $\mathrm{H}_{2} \mathrm{O}_{2}$ than what is required for retention of $50 \%$ viability, indicating other chemical and physical constituents of plasma are required. ${ }^{20}$ This is in agreement with other reports showing that $\mathrm{H}_{2} \mathrm{O}_{2}$ is not the sole plasma effector in various biological stimulations. ${ }^{15,22,23}$ Other possible indicators include change in local temperature at target, UV emission, and resistance of treatment surface, and these may be equally viable candidates for device calibration with a standard unit.

A potential future implementation of a chemical readout could be a diagnostic $\mathrm{H}_{2} \mathrm{O}_{2}$ (or other chemical indicator) strip that changes color and allows evaluation during treatment in the clinic. Strips would need to be calibrated for each plasma device so that each PTU of treatment corresponds to a defined color change. This would then also provide clinicians a common, unifying language for treatment with different plasma systems.

\section{APPLICATION OF PTU TO CURRENT PLASMA SYSTEMS: A TEST CASE}

To test how this approach would translate when we compared another biological readout from the DBD and kINPen, we measured expression of calreticulin (ecto-CRT) on the cell surface after treatment. We have shown that nspDBD treatment of cells at $300 \mathrm{~mJ}$ elicits expression of ecto-CRT, a critical marker for immunogenic cancer cell death. ${ }^{23}$ The measured $\mathrm{H}_{2} \mathrm{O}_{2}$ here was $\sim 24 \mu \mathrm{M}$ which is equivalent to $0.5 \mathrm{PTU}$ for the nspDBD system, based on our definition of PTU described earlier. To determine if 0.5 PTU with a 
kINPen is sufficient to stimulate ecto-CRT expression, we treated cells using parameters sufficient to produce $50 \mu \mathrm{M} \mathrm{H}_{2} \mathrm{O}_{2}$. This corresponds to a 50-second treatment while holding previously defined parameters constant (e.g., voltage, frequency). We observed that kINPen treatment at approximately 0.5 PTU $(60 \mathrm{sec})$ was also able to elicit ecto-CRT. ${ }^{24}$ Although this is only a single example, it demonstrates that the PTU may be suitable for unifying the research data derived from DBD and plasma jet devices.

Calibration of plasma devices based on a common unit like the PTU can accelerate research progress in the plasma medicine field. Researchers rarely use the same plasma device for their studies, because most of these devices are assembled in the lab. This makes it difficult to build upon existing studies, as the first step always requires testing a range of settings that work best for that device. However, if all plasma devices were unified by the PTU, it would provide an indication for which settings to use.

\section{CONCLUSION}

Standardizing a plasma treatment unit (PTU) that unifies DBD and plasma jet devices is undeniably required for clinical acceptance of this technology. Here, we have established that the PTU should not be based on a device setting or plasma property but on a fixed biological readout. The example of cellular metabolic activity as a reflection of plasmainduced toxicity is presented here. Therefore, the PTU is the biological effect from the summation of plasma and treatment parameters. We define 1 PTU as $50 \%$ inhibition of metabolic activity (IC50) of cells, as measured with the live cell metabolic activity assay. $\triangle \mathrm{PTU}$ is merely a repetition of the plasma physics that was used to achieve the $1 \mathrm{PTU}$ effect. This includes all the parameters needed to produce the plasma that elicits IC50. Change in $\mathrm{H}_{2} \mathrm{O}_{2}$ concentration was used here, but other properties such as temperature UV emissions could also be measured to define the increment between PTUs.

We have provided evidence that having a common PTU between devices could also benefit in vitro plasma research, usually the initiation point of all biomedical investigation. Altogether, it is clear that this standard treatment unit is needed to unify and accelerate research in the plasma medicine field, both in the early and late stages.

\section{ACKNOWLEDGMENTS}

SB and KW are supported by grants of the German Federal Ministry of Education and Research (BMBF), Grant Numbers 03Z22DN11 and 03Z22DN12, respectively.

\section{REFERENCES}

1. Kong MG, Kroesen G, Morfill G, Nosenko T, Shimizu T, van Dijk J, Zimmerman JL. Plasma medicine: an introductory review. New J Phys. 2009;11(11):115012.

2. Hirst AM, Frame FM, Arya M, Maitland NJ, O'Connell D. Low temperature plasmas as emerging cancer therapeutics: the state of play and thoughts for the future. Tumour Biol. 2016:37(6): 7021-31.

Volume 8, Issue 2, 2018 
3. Ulrich C, Kluschke F, Patzelt A, Vandersee S, Czaika VA, Bob A, von Hutten J, Hüge R, Kramer A, Assadian O, Lademann J, Lange-Asschenfeldt B. Clinical use of cold atmospheric pressure argon plasma in chronic leg ulcers: a pilot study. J Wound Care. 2015;24(5):196, 198-200, 202-3.

4. Vandamme M, Robert E, Pesnel S, Barbosa E, Dozias S, Sobilo J, Stéphanie L, Le Pape A, Pouvesle JM. Antitumor effect of plasma treatment on U87 glioma xenografts: preliminary results. Plasma Process Polym. 2010;7(3-4):264-73.

5. Keidar M, Shashurin A, Volotskova O, Stepp MA, Srinivasan P, Sandler A, Trink B. Cold atmospheric plasma in cancer therapy. Phys Plasmas. 2013;20(5):057101.

6. Friedman P, Miller V, Fridman G, Lin A, Fridman A. Successful treatment of actinic keratosis using nonthermal atmospheric pressure plasma: a case series. J Am Acad Dermatol. 2017;76(2):349-50.

7. Fridman A, Chirokov A, Gutsol A. Non-thermal atmospheric pressure discharges. J Phys D Appl Phys. 2005;38(2):R1.

8. Lu X, Laroussi M, Puech V. On atmospheric-pressure non-equilibrium plasma jets and plasma bullets. Plasma Sources Sci Technol. 2012;21(3):034005.

9. Kalghatgi S, Kelly CM, Cerchar E, Torabi B, Alekseev O, Fridman A, Friedman G, Azizkhan-Clifford J. Effects of non-thermal plasma on mammalian cells. PloS One. 2011;6(1):e16270.

10. Chernets N, Kurpad DS, Alexeev V, Rodrigues DB, Freeman TA. Reaction chemistry generated by nanosecond pulsed dielectric barrier discharge treatment is responsible for the tumor eradication in the B16 melanoma mouse model. Plasma Process Polym. 2015;12(12):1400-9.

11. Yousfi M, Eichwald O, Merbahi N, Jomaa N. Analysis of ionization wave dynamics in low-temperature plasma jets from fluid modeling supported by experimental investigations. Plasma Sources Sci Technol. 2012;21(4):045003.

12. Isbary G, Heinlin J, Shimizu T, Zimmermann J, Morfill G, Schmidt HU, Monetti R, Steffes B, Bunk W, Li Y, Klaempfl T, Karrer S, Landthaler M, Stolz W. Successful and safe use of 2 min cold atmospheric argon plasma in chronic wounds: results of a randomized controlled trial. Br J Dermatol. 2012;167(2):404-10.

13. Bekeschus S, Schmidt A, Weltmann K-D, von Woedtke T. The plasma jet kINPen - a powerful tool for wound healing. Clin Plasma Med. 2016;4(1):19-28.

14. Dobrynin D, Friedman G, Fridman A, Starikovskiy A. Inactivation of bacteria using DC corona discharge: role of ions and humidity. New J Phys. 2011;13(10):103033.

15. Dobrynin D, Fridman G, Friedman G, Fridman A. Physical and biological mechanisms of direct plasma interaction with living tissue. New J Phys. 2009;11(11):115020.

16. Kalghatgi S, Friedman G, Fridman A, Clyne AM. Endothelial cell proliferation is enhanced by low dose non-thermal plasma through fibroblast growth factor-2 release. Ann Biomed Eng. 2010;38(3):748-57.

17. Steinbeck MJ, Chernets N, Zhang J, Kurpad DS, Fridman G, Fridman A, Freeman TA. Skeletal cell differentiation is enhanced by atmospheric dielectric barrier discharge plasma treatment. PLoS One. 2013;8(12):e82143.

18. Lee A, Lin A, Shah K, Singh H, Miller V, Rao SG. Optimization of non-thermal plasma treatment in an in vivo model organism. PLoS One. 2016;11(8):e0160676.

19. Rupf S, Lehmann A, Hannig M, Schäfer B, Schubert A, Feldmann U, Schindler A. Killing of adherent oral microbes by a non-thermal atmospheric plasma jet. J Med Microbiol. 2010;59(2):206-12.

20. Bekeschus S, Lin A, Fridman A, Wende K, Weltmann K-D, Miller V. A comparison of floating-electrode DBD and kINPen jet: plasma parameters to achieve similar growth reduction in colon cancer cells under standardized conditions. Plasma Chem Plasma Process. 2018;38(1):1-12.

21. Lu X, Naidis G, Laroussi M, Reuter S, Graves D, Ostrikov K. Reactive species in non-equilibrium atmospheric-pressure plasmas: generation, transport, and biological effects. Phys Rep. 2016;630:1-84.

22. Lin A, Chernets N, Han J, Alicea Y, Dobrynin D, Fridman G, Freeman TA, Fridman A, Miller V. Non-equilibrium dielectric barrier discharge treatment of mesenchymal stem cells: charges and reactive oxygen species play the major role in cell death. Plasma Process Polym. 2015;12(10):1117-27. 
23. Lin A, Truong B, Patel S, Kaushik N, Choi EH, Fridman G, Fridman A, Miller V. Nanosecond-pulsed DBD plasma-generated reactive oxygen species trigger immunogenic cell death in A549 lung carcinoma cells through intracellular oxidative stress. Int J Mol Sci. 2017;18(5):E966.

24. Bekeschus S, Mueller A, Gaipl U, Weltmann K-D. Physical plasma elicits immunogenic cancer cell death and mitochondrial singlet oxygen. IEEE Trans Rad Plasma Med Sci. 2017;2(2):136-46.

Volume 8, Issue 2, 2018 
